\title{
Editorial \\ Lorentz Violation in Astroparticles and Gravitational Waves
}

\author{
Marco Schreck (iD)
}

check for

updates

Citation: Schreck, M. Lorentz

Violation in Astroparticles and Gravitational Waves. Galaxies 2022, 10, 13. https://doi.org/10.3390/ galaxies10010013

Received: 5 January 2022

Accepted: 11 January 2022

Published: 17 January 2022

Publisher's Note: MDPI stays neutral with regard to jurisdictional claims in published maps and institutional affiliations.

Copyright: (C) 2022 by the author. Licensee MDPI, Basel, Switzerland. This article is an open access article distributed under the terms and conditions of the Creative Commons Attribution (CC BY) license (https:// creativecommons.org/licenses/by/ $4.0 /)$.
Physics Department, Federal University of Maranhão, Bacanga Campus, São Luís 65080-805, Brazil; marco.schreck@ufma.br

Lorentz invariance is one of the fundamental continuous symmetries of the laws of nature. On the one hand, the Standard Model of elementary particles exhibits global Lorentz invariance. On the other hand, General Relativity can be formulated in a way that makes local Lorentz invariance explicit. Hence, the importance of this fundamental symmetry in the current physical paradigm is evident. One possibility of searching for physics beyond the Standard Model and General Relativity is to conceive experiments capable of detecting possible minuscule violations of Lorentz symmetry. Since such violations were demonstrated to occur in particular string field theories [1,2] as well as in loop quantum gravity [3], tests of Lorentz invariance provide a window into Planck scale physics, which makes this field one of the central thrusts of high-energy physics in the 21st century.

This Special Issue covers state-of-the-art research on Lorentz violation in the settings of gamma rays, ultra-high-energy cosmic rays, astrophysical neutrinos as well as the production and propagation of gravitational waves, among other topics. A subset of the compiled papers employs the language of the Standard-Model Extension (SME) [4-10]. The latter is a comprehensive effective field theory framework that parametrizes all deviations from Lorentz invariance that are scalars under coordinate transformations and respect the gauge structure of the Standard Model. The absence of experimental signals for Lorentz violation translates into constraints on SME coefficients quantifying the extent to which Lorentz invariance can be considered as an established spacetime property. The SME decomposes into a matter sector [4,6-9] and a gravitational sector [5,10]. The minimal SME involves field operators of mass dimensions $d=3$ and 4 [4,5], whereas the nonminimal SME incorporates field operators of arbitrary mass dimensions [6-10].

The papers published in this Special Issue provide novel, interesting phenomenological and theoretical contributions to this hot topic. Any type of Lorentz violation introduced at the level of effective field theory can somehow be mapped to the SME; however, the mapping of a specific model may not be obvious at the first place. The articles [11-13] provide SME-based analyses and reviews, respectively. The papers [14-18] study phenomena associated with Lorentz violation in specific models and the authors of Refs. $[15,18]$ establish connections of their models to the SME.

In particular, the analysis performed in Ref. [11] is based on tensor-valued background coefficients of the minimal SME gravity sector, which are denoted as $s^{\mu v}$. Within the regime of Newtonian gravity the impact of the background field on the rotation of ellipsoidal neutron stars is explored. The rotating star produces gravitational waves and the metric perturbations of both polarizations are numerically obtained. The smoking gun for Lorentz violation is a two-fold precession, which should give rise to a clear signal in the gravitational-wave spectrum. The paper [12] provides an analysis of gravitational-wave propagation subject to generic SME background fields contracted with field operators of minimal and nonminimal mass dimensions. In particular, configurations known as "aether-like" are studied, which can be expressed in terms of constant vector-valued background fields. The authors find that dispersion relations of gravitational waves remain unaffected by Lorentz violation in these cases. They also conceive a method to construct generic gauge-invariant modifications of the Einstein-Hilbert action in the regime of weak 
gravitational fields. Thus, since Ref. [11] focuses on the production and Ref. [12] on the propagation of gravitational waves in the presence of Lorentz-violating background fields, both papers cover complementary physics.

Reference [13] provides a review of a specific part of the matter sector where gravity plays a minor role: neutrinos. It summarizes the basics of the SME neutrino sector, the formalism incorporating Lorentz violation in neutrinos as well as its implications on physical effects such as neutrino oscillations. Furthermore, the paper gives an overview of different types of experiments involving reactor, solar and astrophysical neutrinos, respectively. Finally, sensitivities on Lorentz violation in neutrinos are discussed.

The author of Ref. [14] focuses on a cosmology whose scale factor exhibits stochastic oscillations. Such a property is considered in some theories to avoid the necessity of a cosmological constant or dark energy for stages of an accelerated expansion of the Universe. An averaging procedure gives rise to nonstandard contributions in Newtonian dynamics, in particular, an additional term in the radial equations of motion of a test particle in the gravitational field of a central body. The latter modification is demonstrated to be capable of explaining certain characteristics in galactic rotation curves without the need of dark matter. The analysis of Ref. [15] takes place within the scenario of a preferred frame where the one-way speed of light is isotropic. In contrast, the speed of light turns anisotropic in all other inertial frames that move with respect to the preferred one. A modified infinitesimal path length interval squared leads to the action of a single, massive particle influenced by Lorentz violation. Performing a specific coordinate transformation provides a set of modified Maxwell equations, i.e., the dynamics of electromagnetic fields is also effected. The modifications can be mapped to a subset of the $k_{F}$ coefficients parametrizing the minimal, $C P T$-even, nonbirefringent SME photon sector. Certain particle physics processes such as the GZK process and pair production from photons are investigated and the implications on the cosmic-ray spectrum are elucidated. Reference [16] is dedicated to a particular class of isotropic modified dispersion equations for massive particles. In the setting of Finsler geometry it is possible to establish a connection to a modified theory of spin-1/2 fermions, which can be generalized to a curved spacetime. Studying the kinematics of the $\Delta$ resonance production, which plays a role for the GZK cutoff, allows for obtaining a tight constraint on the deformation functions in the nonstandard dispersion equation proposed initially. Furthermore, the altered attenuation length of high-energy protons is investigated, too.

The authors of Ref. [17] are interested in constraining a modification of the photon group velocity depending on both the frequency and polarization. The latter can be identified with an isotropic, dimension- 5 contribution of the SME photon sector, which also implies vacuum birefringence. Data on the linear polarization of light acquired from a set of Active Galactic Nuclei form the base of a spectropolarimety analysis. By doing so, an anomalous change of the polarization angle of light, which may be a consequence of Lorentz violation, is searched for in the data. The analysis leads to a novel constraint on vacuum birefringence described by an operator of nonminimal mass dimension. Finally, Ref. [18] delves into a scenario of a fifth spacetime dimension, which is a generalization of Kaluza-Klein theory known as Space-Time-Matter theory. In this approach, the extra coordinate is not assumed to be closed or compact. The velocity associated with the fifth coordinate is related to a change of particle masses in time. Such variations can be driven in an expanding universe by the presence of a particular $c$-type coefficient in the minimal-SME Dirac fermion sector. Due to a special constraint in protons, a properly normalized variation of the proton mass in time is concluded to be smaller than $10^{-6}$ times the cosmological expansion rate. The latter number is not unnaturally small in this context and future experiments are likely to improve that limit further.

To summarize, the papers compiled in this Special Issue present interesting results and complement the searches for Lorentz violation in astroparticles and gravitational waves already existing in the literature. As analyses based on the SME as well as beyond the SME 
are contained in the Special Issue, interaction between these two different approaches of quantum gravity phenomenology is expected to be fostered.

Funding: M.S. is indebted to FAPEMA Universal 00830/19, CNPq Produtividade 312201/2018-4, and CAPES/Finance Code 001 for financial support.

Acknowledgments: M.S. appreciates the support by the editors and staff of Galaxies that enabled the successful completion of this Special Issue.

Conflicts of Interest: The author declares no conflict of interest.

\section{References}

1. Kostelecký, V.A.; Samuel, S. Spontaneous Breaking of Lorentz Symmetry in String Theory. Phys. Rev. D 1989, 39, 683. [CrossRef] [PubMed]

2. Kostelecký, V.A.; Potting, R. Expectation Values, Lorentz Invariance, and CPT in the Open Bosonic String. Phys. Lett. B 1996, 381, 89. [CrossRef]

3. Bojowald, M.; Morales-Técotl, H.A.; Sahlmann, H. Loop Quantum Gravity Phenomenology and the Issue of Lorentz Invariance. Phys. Rev. D 2005, 71, 084012. [CrossRef]

4. Colladay, D.; Kostelecký, V.A. Lorentz-Violating Extension of the Standard Model. Phys. Rev. D 1998, 58, 116002. [CrossRef]

5. Kostelecký, V.A. Gravity, Lorentz Violation, and the Standard Model. Phys. Rev. D 2004, 69, 105009. [CrossRef]

6. Kostelecký, V.A.; Mewes, M. Electrodynamics with Lorentz-Violating Operators of Arbitrary Dimension. Phys. Rev. D 2009, 80, 015020. [CrossRef]

7. Kostelecký, V.A.; Mewes, M. Neutrinos with Lorentz-Violating Operators of Arbitrary Dimension. Phys. Rev. D 2012, 85, 096005. [CrossRef]

8. Kostelecký, V.A.; Mewes, M. Fermions with Lorentz-Violating Operators of Arbitrary Dimension. Phys. Rev. D 2013, 88, 096006. [CrossRef]

9. Kostelecký, V.A.; Li, Z. Gauge Field Theories with Lorentz-Violating Operators of Arbitrary Dimension. Phys. Rev. D 2019, 99, 056016. [CrossRef]

10. Kostelecký, V.A.; Li, Z. Backgrounds in Gravitational Effective Field Theory. Phys. Rev. D 2021, 103, 024059. [CrossRef]

11. Xu, R.; Gao, Y.; Shao, L. Signatures of Lorentz Violation in Continuous Gravitational-Wave Spectra of Ellipsoidal Neutron Stars. Galaxies 2021, 9, 12. [CrossRef]

12. Nascimento, J.R.; Petrov, A.Y.; Vieira, A.R. On Plane Wave Solutions in Lorentz-Violating Extensions of Gravity. Galaxies 2021, 9 , 32. [CrossRef]

13. Roberts, Á. Astrophysical Neutrinos in Testing Lorentz Symmetry. Galaxies 2021, 9, 47. [CrossRef]

14. Smolyaninov, I.I. Oscillating Cosmological Force Modifies Newtonian Dynamics. Galaxies 2020, 8, 45. [CrossRef]

15. Burde, G.I. Lorentz Violation by the Preferred Frame Effects and Cosmic and Gamma Ray Propagation. Galaxies 2021, 9, 119. [CrossRef]

16. Torri, M.D.C. Quantum Gravity Phenomenology Induced in the Propagation of UHECR, a Kinematical Solution in Finsler and Generalized Finsler Spacetime. Galaxies 2021, 9, 103. [CrossRef]

17. Zhou, Q.-Q.; Yi, S.-X.; Wei, J.-J.; Wu, X.-F. Constraints on Lorentz Invariance Violation with Multiwavelength Polarized Astrophysical Sources. Galaxies 2021, 9, 44. [CrossRef]

18. Overduin, J.; Ali, H.; Walz, F. Constraints on Space-Time-Matter Theory in the Framework of the Standard-Model Extension. Galaxies 2021, 9, 26. [CrossRef] 